Musées, Patrimoine et Culture scientifiques et techniques

$145 \mid 2013$

janvier-février 2013

\title{
L'expérience PLUG-Université Paris-Nuit : un juste équilibre entre apprentissage et jeu
}

Coline Aunis, Isabelle Astic, Éric Gressier et Areti Damala

\section{OpenEdition \\ Journals}

Édition électronique

URL : http://journals.openedition.org/ocim/1169

DOI : $10.4000 /$ ocim. 1169

ISSN : 2108-646X

Éditeur

OCIM

Édition imprimée

Date de publication : 1 janvier 2013

Pagination : 5-12

ISSN : 0994-1908

Référence électronique

Coline Aunis, Isabelle Astic, Éric Gressier et Areti Damala, «L'expérience PLUG-Université Paris-Nuit :

un juste équilibre entre apprentissage et jeu », La Lettre de I'OCIM [En ligne], 145 | 2013, mis en ligne le

01 janvier 2015, consulté le 02 mai 2019. URL : http://journals.openedition.org/ocim/1169 ; DOI :

10.4000/ocim. 1169 


\title{
L'expérience PLUG-Université Paris-Nuit : un juste équilibre entre apprentissage et jeu
}

\author{
Coline Aunis, Isabelle Astic, Éric Gressier \\ et Areti Damala *
}

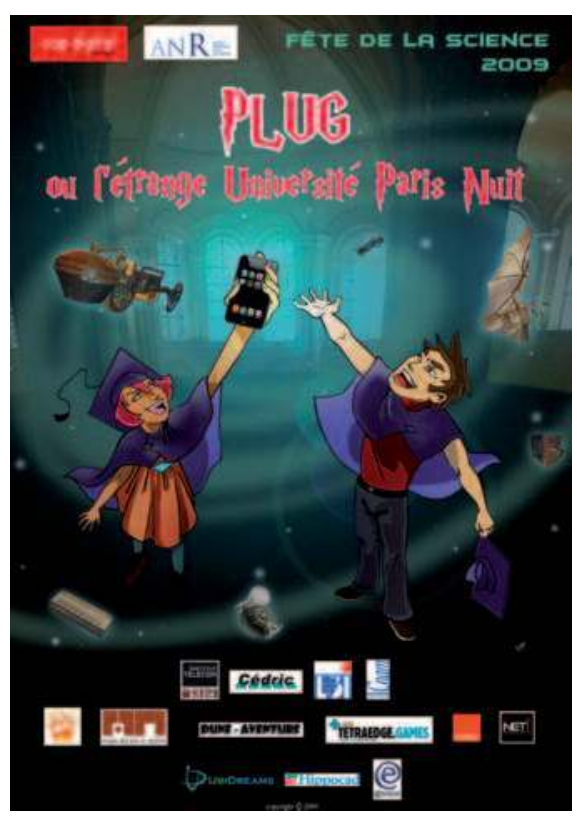

Un des deux jeux déployés dans le cadre du projet PLUG.

(c) PLUG

* Coline Aunis est chef de projets web et multimedia au musée des Arts et Métiers coline.aunis@cnam.fr Isabelle Astic est responsable des collections Informatique et Réseaux au musée des Arts et Métiers

Éric Gressier est professeur au CNAM et chercheur au centre d'Étude et de Recherches en

Informatique et Communications

(CEDRIC, équipe SEMpIA), CNAM

Areti Damala est post-doctorante et chef de projets en Informatique et Ingénierie Culturelle au centre d'Étude et de Recherches en Informatique et Communications, CNAM
$\grave{A}$ travers la description des principes et de la mise en œuvre de la seconde étape du jeu pervasif PLUG, les responsables du projet analysent les perspectives de son utilisation comme outil pédagogique permettant d'aller plus loin dans l'expérience de visite ainsi que les possibilités offertes à l'institution muséale dans sa réflexion sur les nouvelles formes de la médiation.

\section{Introduction}

Le projet PLUG (PLay Ubiquitous Games and play more) était un projet supporté par l'ANR (Agence Nationale de la Recherche). Il a duré 27 mois, de janvier 2008 à mars 2010, et correspond à un budget de $400 \mathrm{~K} €$ environ. Deux types de partenaires constituaient le consortium : des partenaires académiques (l'université de La Rochelle-L3i, l'institut Telecom et le Conservatoire national des Arts et Métiers), des industriels (le studio de jeux vidéo TetraEdge, Orange) et une association (Dune Aventures). L'objectif du projet était d'étudier de nouvelles façons d'interagir avec un musée, de valoriser ses contenus et d'élaborer une médiation innovante à travers une approche jeux pervasifs/serious games qui combinerait harmonieusement pédagogie et plaisir de jouer. Le consortium reflétait la diversité des compétences nécessaires : évaluation des usages, design d'interaction, design de lieux, game design, scénarisation de jeux grandeur nature, adaptabilité du game design en fonction du joueur, patrimoine et contenus numériques, médiation culturelle, approche opérateur de réseaux mobiles et services associés, systèmes informatiques mobiles. 
Le projet PLUG a conçu et mis en œuvre deux jeux pervasifs : PLUG-Les Secrets du Musée (PSM) (Astic et Aunis, 2009) et PLUG-Université Paris-Nuit (PUPN). Chaque année du projet a été consacrée à l'élaboration et à l'expérimentation d'un jeu. PSM a pleinement bénéficié d'une étude d'usage intensive car il a continué à être expérimenté lors de la deuxième année du projet (Gentès, 2009). Au total, environ 700 personnes, dont 250 élèves de primaire, ont joué à PSM sur les deux années. Les dernières expériences se sont déroulées sans l'assistance des partenaires techniques.

La question principale à laquelle l'équipe de conception du jeu souhaitait apporter un élément de réponse était : comment mettre l'approche pédagogique plus au cour du jeu ? L'analyse du premier projet PSM nous a montré que la volonté de présenter le musée et de valoriser les objets était omniprésente et prioritaire mais que le jeu avait été conçu en premier lieu à travers son format et ses règles ( 7 familles) avant de définir la place des éléments de contenus. Pour le second projet PUPN, nous avons donc choisi d'étudier une approche plus marquée par la pédagogie : nous l'avons construit de façon inverse en définissant d'abord la trame pédagogique, puis en établissant le jeu et la scénarisation qui l'englobe.

Cet article propose dans sa première partie une décription des principes de game design adoptés dans le but de permettre à la fois un apprentissage et un amusement. La seconde partie traite de la mise en œuvre de ces principes. La conclusion dresse un bilan de notre contribution au domaine et présente quelques perspectives.

\section{PLUG-Université Paris-Nuit : priorité à la pédagogie}

\section{Pédagogie versus plaisir de jouer:}

une question d'adaptabilité

Suite aux retours d'expérience du premier jeu PSM, la question de fond «comment trowver le juste équilibre entre jeu et apprentissage, plaisir et pédagogie, sans nuire à la qualité de l'expérience du visiteur-joueur? » s'est dégagée. Corollaire de cette question : comment moduler le jeu en fonction des compétences et de l'engagement du joueur ? Ceci a mené à la fonction d'adaptabilité, mise en œuvre par le L3i-La Rochelle, qui a permis d'adapter le contenu du jeu au profil du joueur.

Lorsque l'on travaille sur un serious game, le juste équilibre entre le serious et le game est difficile à trouver.
La frontière entre le ludique et l'apprentissage n'est pas clairement matérialisée. C'est d'autant plus complexe à déterminer que cette frontière n'est pas univoque : tout dépend également du joueur, son profil, son âge et ses attentes. Comment s'assurer que le jeu et l'apprentissage se complètent l'un l'autre à proportions égales pour un nombre maximum de joueurs? Comment être sûr que le joueur s'est amusé tout en apprenant ? Il n'existe pas de solution répertoriée prête à l'emploi. Ce sont les retours suite à PSM qui nous ont permis de nous rendre compte que l'aspect jeu avait pris le dessus sur l'apprentissage de la collection. Il fallait donc faire en sorte que l'accès au contenu s'effectue de façon imperceptible mais évidente, complètement immergée dans le jeu. Il fallait également que cela se fasse sans effort particulier pour éliminer tout découragement d'un joueur.

L'adaptabilité du jeu au profil des joueurs eux-mêmes semblait être l'une des clés incontournables de notre approche. Cette fonctionnalité de la plateforme pouvait permettre un apprentissage tel que nous le souhaitions : sur mesure et progressif. Conséquence immédiate de ce choix : le moteur d'adaptabilité dans PUPN devait s'appuyer sur un contenu conçu avec une trame pédagogique vissée à la scénarisation.

Ainsi, et pour s'adresser à un maximum de profils aux connaissances techniques/scientifiques et historiques/ sociologiques variées, l'équipe de conception a développé deux types de parcours de jeu : un parcours scientifique et technique, et un parcours historique et sociologique. L'autre clef de réussite était la trame pédagogique qui devait soutenir la scénarisation et le game design de notre jeu pervasif.

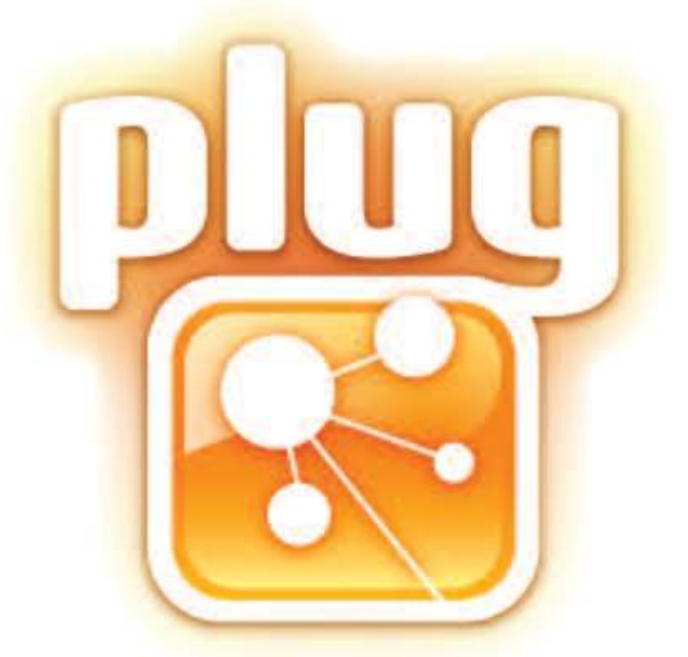


Le moteur d'adaptabilité produit par le L3i-La Rochelle a été construit en s'appuyant sur des contenus adaptés à différents profils de joueurs. Le moteur d'adaptabilité se base principalement sur la progression des équipes qui sont suivies « à la trace »; la pervasivité du jeu, l'imbrication entre le monde du jeu et le monde réel. Il permet en effet de rendre compte du parcours précis des joueurs grâce aux tags RFID répartis sur l'ensemble des salles du musée et avec lesquels les joueurs interagissent continuellement. La progression dans le jeu, concrétisée par l'action de lire ou encore de taguer les spots RFID, est ainsi plus ou moins rapide si le joueur est à l'aise avec les technologies utilisées et s'il possède des acquis et connaissances pour suivre les indices ou répondre rapidement aux questions.

Les équipes avancent en parallèle. Les indices et questions posées présentent trois niveaux de difficulté : facile, moyen, difficile. Au bout de 5 minutes d'absence de réponse de la part d'une équipe, le moteur d'adaptabilité envoie un indice de niveau inférieur afin de faciliter la recherche et de ne pas décourager les joueurs. Cela étant, l'indice suivant proposé est automatiquement " réhaussé » d'un niveau de difficulté afin de stimuler au maximum l'implication des joueurs. En parallèle la fonction maître du jeu (MdJ) permet de suivre les parcours respectifs de chacune des équipes en temps réel qui sont ainsi comparées en direct, et en coulisse, par les membres du projet. L'un des objectifs est de faire avancer les équipes au même rythme pour qu'elles se rencontrent toutes lors de la scène finale. En fonction de la progression des équipes, et par l'intermédiaire du moteur d'adaptabilité, le MdJ peut modifier la difficulté des parcours : l'envoi d'indices de moindre difficulté permet d'accélérer les équipes les plus lentes, et à l'inverse, l'envoi d'indices difficiles ou d'énigmes supplémentaires ralentit certaines équipes trop rapides. Ce double suivi optimise l'engagement des joueurs individuellement et collectivement, et permet de cadrer à la théorie du flow (Csikszentmihalyi, 1990) (1).

\section{Définition de la trame pédagogique}

Pour la seconde édition du jeu, notre but était d'expliquer aux visiteurs-joueurs qu'une innovation n'apparaît pas ex-nihilo mais qu'elle est issue d'une chầne d'innovations réalisées en amont et n'appartenant pas forcément au même domaine scientifique et technique. Par exemple, la structure de l'hydroptère, bateau volant sur les eaux, a été imaginée à partir des études et constatations faites sur les procédés de déformation

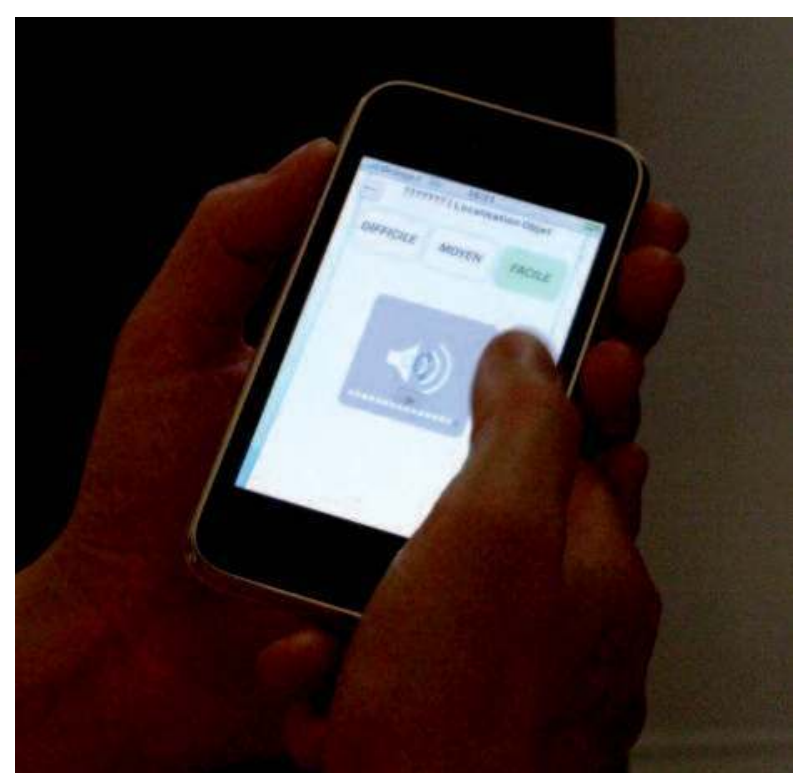

Le moteur d'adaptabilité de PUPN permettait d'ajuster le niveau des indices et questions aux profils des joueurs.

(c) PLUG

des charpentes de bâtiments. Il nous paraissait également important de montrer qu'une innovation naît toujours dans un contexte favorable à son éclosion, qu'il soit social, politique et/ou économique. Par exemple, l'essor des horloges marines au XVIIIe siècle est dû au développement du commerce maritime et la nécessité pour l'équipage de connaître sa position en permanence et de façon de plus en plus précise. Pour ce faire, nous devions choisir des objets conçus à partir de nombreux procédés techniques; notre choix s'est porté sur l'hydroptère et le téléphone portable, tous deux d'une haute technicité. Dans la suite du document, nous les appellerons « objets cibles».

Pour chacun des objets-cibles, nous avons déterminé quatre innovations antérieures présentes au musée, sans lesquelles ils n'auraient pas pu voir le jour et que nous appellerons « objets intermédiaires ». Le lien entre l'« objet cible » et les « objets intermédiaires » peut être direct : le procédé technique ou la recherche scientifique mis en œuvre pour l'objet intermédiaire est intégré directement dans l'objet cible (par exemple, le téléphone portable existe grâce à la maîtrise de la technologie du silicium) ou indirect : un objet intègre le fruit de recherche issu d'un autre domaine scientifique/technique que le sien. Dans notre cas, l'hydroptère a pu être conçu grâce aux recherches effectuées en hydrodynamisme, science issue de l'aérodynamisme datant elle-même du début du XXe siècle. L'objet-cible « hydroptère » a donc été 
relié aux objets intermédiaires « avion de Clément Ader » (premier avion à se soulever du sol - et donc à voler - dont la forme avait été conçue à partir de l'étude du vol d'une chauve-souris) et «Blériot XI » (premier avion à traverser la Manche, dont les formes préfiguraient les premières recherches en aérodynamisme). Cette comparaison permettait de montrer l'importance de l'aérodynamisme en tant que science mais aussi le rôle fondamental de la portance en tant que procédé technique utilisé pour les avions et l'hydroptère.

Deux parcours ont été conçus, chacun reliant 5 objets, et pouvant être parcourus selon un axe plutôt technologique ou plutôt historique pour présenter le contexte historique propice à la naissance de l'innovation (Astic, 2011).

\section{Un apprentissage confirmé}

Intégrer une trame pédagogique au cœur du jeu ne nous semblait pas suffire. Nous voulions également que la dynamique propre du jeu favorise l'assimilation des connaissances. Le visiteur-joueur devait devenir un véritable acteur de son apprentissage sans qu'il ait à fournir un effort mesuré et conscient. Nous souhaitions que le jeu soit le médiateur proprement dit. Pour cela, nous avons déployé deux stratégies : d'une part, les joueurs devaient rechercher activement les solutions aux questions posées, d'autre part, leurs réponses étaient systématiquement évaluées par le système et validées ou refusées.

Pour les joueurs, la recherche des réponses a été physiquement et intellectuellement active. La trame du scénario et la volonté d'utiliser les RFID ont amené à concevoir un jeu où le visiteur-joueur explore le musée à la recherche d'objets que des indices lui permettent de localiser. Le format de PPUN correspondait donc à celui du jeu de piste, rendant ainsi le joueur actif tant par sa participation physique qu'intellectuelle et ce, à son insu.

Pour chaque « objet intermédiaire », une question technique, scientifique ou historique était posée en lien avec l'« objet cible ». Pour développer un apprentissage actif, notre objectif était que le joueur n'ait pas simplement à choisir des solutions préparées mais qu'il dispose de documentation lui permettant de répondre à toute question posée sur un objet. De la riche documentation multimédia était disponible dans le terminal mis à la disposition des joueurs (un iPhone) ou au centre de documentation du musée.

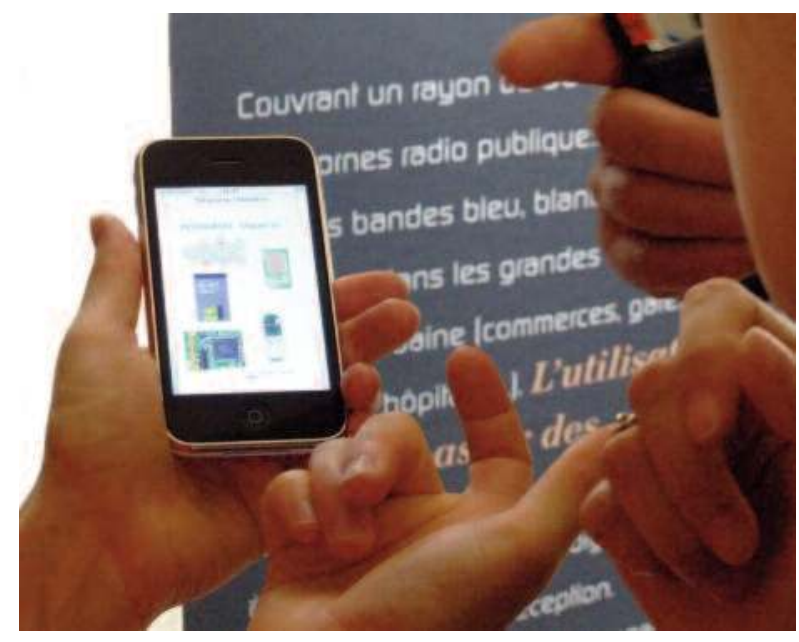

Une riche documentation est disponible dans le smartphone et au centre de documentation du musée. (c) PLUG

La solution proposée par les joueurs était immédiatement vérifiée. Si elle était fausse, la question était reposée. Une bonne réponse permettait de passer à l'objet suivant. Cette phase de validation était importante. Elle était la récompense à la recherche sur la compréhension de l'objet. Toutefois, une bonne réponse, non comprise pour autant par le visiteurjoueur, n'était pas à exclure. C'est pourquoi, PUPN s'achevait sur une scène de restitution finale, où chaque équipe devait présenter le résultat de ses travaux, et notamment expliquer les liens entre les différents « objets intermédiaires » et l'« objet cible » qui leur avait été assigné.

Cette formalisation à l'oral devant un jury évaluateur intégré à la scénarisation permettait d'ancrer les connaissances acquises et nous fournissait, à nous concepteurs, les moyens de vérifier le niveau de compréhension des visiteurs-joueurs, voire de donner les compléments d'explications nécessaires. D'autre part, le jeu étant divisé en 4 parcours différents (axe technique/historique, hydroptère/téléphone portable), cela offrait une opportunité pour que les connaissances acquises par les uns soient communiquées aux autres. Nous avions trouvé un moyen efficace d'immerger l'évaluation pédagogique dans l'univers du jeu sans que cela ne soit perçu comme tel et que ça pèse sur le plaisir de jouer.

\section{La muséologie intégrée au gameplay}

Dans le cadre du projet, les personnels du musée souhaitaient pousser l'apprentissage des joueurs plus loin que la simple appropriation des collections. Nous voulions que l'intérêt du jeu se porte aussi sur le musée 
en tant que lieu d'exposition et de conservation. Nous avons ainsi structuré le gameplay autour de la recherche des domaines de collections («Instruments scientifiques », « Matériaux », «Construction », « Communication », «Énergie », « Transport », «Église ») et la recherche d'objets dans ces différents espaces. Les étapes de recherche des domaines de collections dans le jeu mettent en avant le travail muséographique essentiel à une présentation réfléchie des collections et leur accessibilité. Les étapes de recherche de l'époque de l'objet (avant 1750, de 1750 à 1850, de 1850 à 1950, après 1950) pointent la structuration de l'espace et la cohérence apportée à la présentation des collections d'un même domaine. Enfin, nous tenions à ce que les joueurs retrouvent et taguent les objets en utilisant les numéros d'inventaire, véritables témoins du travail de conservation, préservation et répertoriation des objets dans le cadre d'un musée.

\section{Dynamiser le jeu et créer du lien social entre les équipes}

Des rencontres impromptues entre équipes ont été imaginées pour consolider le lien social entre les visiteurs-joueurs. Créer des occasions de rencontres maintient une équipe dans le rythme du jeu et évite le risque d'un jeu trop monotone. Par exemple, lorsqu'une équipe était en retard, le Maître du Jeu la convoquait ainsi qu'une autre pour qu'elles s'affrontent dans le cadre d'une joute. Chaque membre de chaque équipe mimait un objet du musée sans que les autres participants, y compris ceux de son équipe, le connaissent. L'équipe ayant reconnu le plus d'objets gagnait la partie et le droit de pirater le cartable

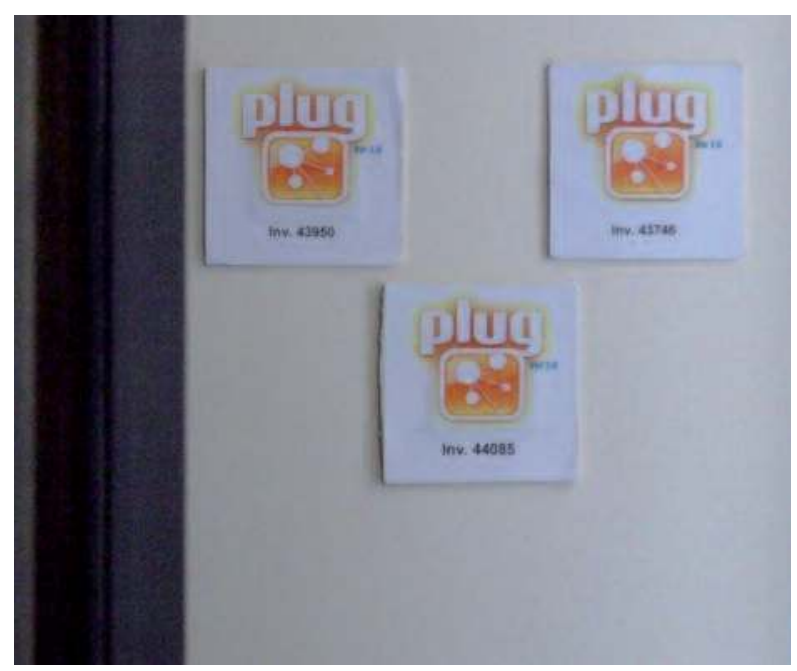

Chaque objet était identifié par son numéro d'inventaire sur les tags RFID. (C) PLUG de l'autre afin d'y récupérer du contenu ! Cette épreuve permettait ainsi de forger un esprit d'équipe.

\section{Mise en œuvre de PLUG-Université Paris-Nuit}

\section{Un véritable univers de jeu}

Même si nous souhaitions renforcer la notion d'apprentissage dans le cadre de PUPN, il ne fallait pas négliger l'aspect ludique : PLUG est un jeu qui s'inscrit de fait dans un game design et un gameplay. Nous avons réfléchi à une quête dans le musée qui avait l'avantage d'être ludique, mais l'aspect linéaire et traditionnel des indices et des questions nous paraissait trop sérieux et ennuyeux. Il fallait pouvoir compter sur des rebondissements extérieurs dans le but de donner du souffle et du rythme pour tenir les joueurs en alerte.

L'association Dune Aventures, dont le domaine de compétence est la scénarisation et la mise en place de jeux grandeur nature en milieu urbain, a pris une part active dans l'élaboration du jeu PPUN. Elle a apporté une dimension de jeu de rôle qui a renforcé l'aspect ludique. Elle a proposé une dimension scénaristique au jeu, par l'intermédiaire de comédiens et le déploiement d'un véritable univers de jeu.

D’après le scénario, une université secrète nommée UPN (Université Paris-Nuit) agit la nuit au sein du musée en soumettant de potentiels étudiants à de nombreuses épreuves. Le but de cette université secrète est de recruter une élite qui s'inspirerait des inventions présentées dans le musée pour réfléchir et mettre en place de nouvelles réalisations grandioses et propices au bien de l'humanité.

\section{Scénario et comédiens}

Les joueurs sont les potentiels étudiants de l'UPN mais ils ne le savent pas au préalable. En arrivant au musée, ils sont accueillis par un guide qui s'avère être un commissaire de police. Celui-ci leur montre un tract déchiré sur lequel on peut lire «... les épreuves d'admission commencent ce jour même... ». Le commissaire leur demande donc d'infiltrer cette organisation mystérieuse afin d'en savoir plus sur ses agissements. Voilà donc nos joueurs enrôlés comme policiers infiltrés ! Le commissaire les fait entrer par une porte dérobée en leur donnant rendez-vous plus tard à leur sortie du musée une fois leur mission accomplie. Derrière cette porte dérobée, les joueurs se retrouvent avec M. Picard, le surveillant général de l'université secrète. Il les accueille tels des candidats désireux d'intégrer l'UPN et les met en garde des 


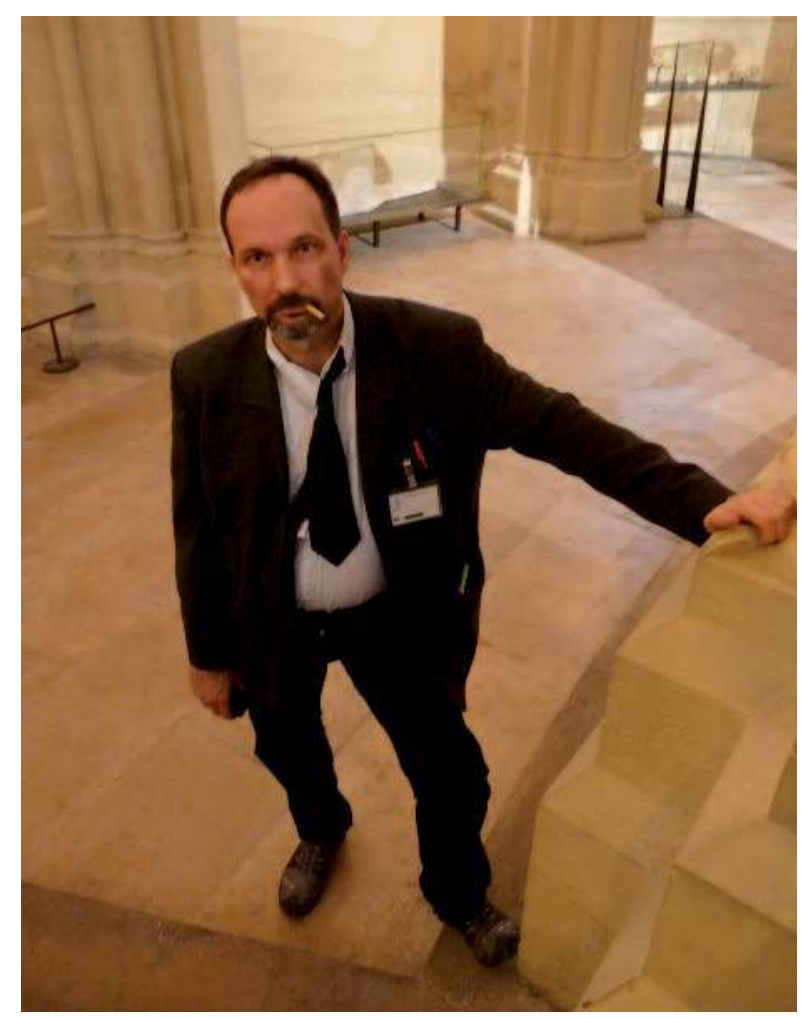

L'univers de PUPN : des comédiens dans des lieux insolites (c) PLUG

multiples épreuves à passer pour cela, avec notamment une épreuve finale d'envergure face à la Directrice. Puis la Grande Z, présidente de l'incontournable Bureau des Élèves, les accueille de façon bienveillante tout en leur remettant l'ensemble des équipements dont ils auront besoin pour passer les épreuves, et qui composent le «cartable électronique ». Elle leur explique également la joute, une tradition officieuse et spécifique à l'université.

Lors de la scène finale, les joueurs font face à la Grande Z, M. Picard et la Directrice de l'UPN. Cette dernière leur raconte la genèse et les objectifs de cette université parallèle : former les meilleurs étudiants qui soient pour en faire des inventeurs de génie dont l'inspiration se nourrirait des inventions passées. Chaque équipe est appelée à la barre d'un tribunal pour répondre à l'ultime énigme : restituer leur parcours de jeu en résumant le lien entre l'objet contemporain (l'objet cible) et l'un des objets ancêtres (objets intermédiaires). Chacun doit enfin argumenter son désir d'intégrer l'UPN. La directrice est seule juge d'admettre ou non les différents candidats à l'université, après avoir pris conseils et avis auprès de la Grande Z. Avant que tout le monde ne se sépare, la Directrice fait prêter serment à tous les joueurs de ne jamais dévoiler le secret de l'UPN et d'utiliser la

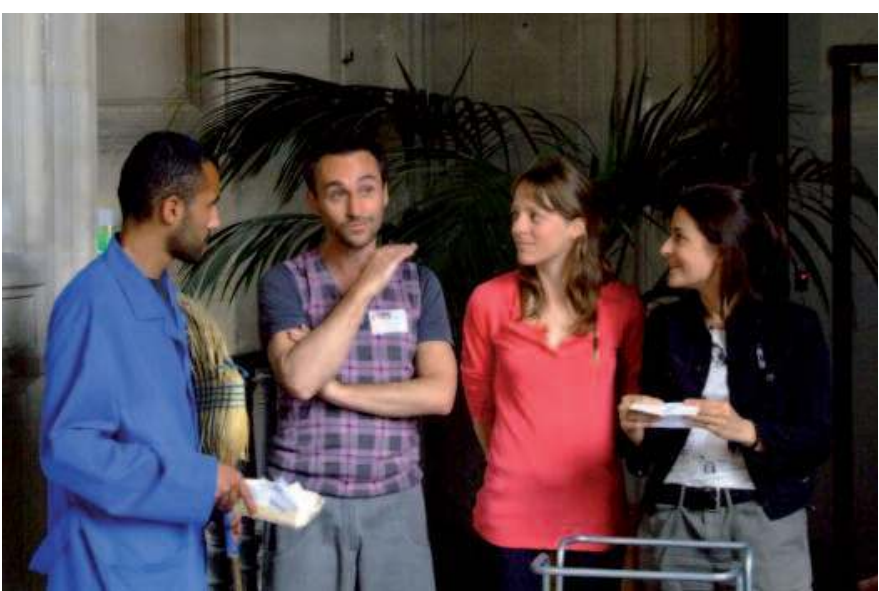

La restitution des connaissances acquises par les joueurs lors de la grande scène finale.

() PLUG

couverture suivante : UPN est une société d'audioguide signifiant Univers Pédagogique Nouveau. À leur sortie, les joueurs sont cueillis par le commissaire de police qui leur demande un rapport suite à leur mission : "Qu'est-ce que l'UPN ? ". Dans la très grande majorité des cas, aucun joueur ne trahit l'UPN.

En ce qui concerne l'univers du jeu, les responsables du projet ont travaillé à immerger les joueurs dans un décor et une ambiance qui rappellent Harry Potter et l'école de Poudlard. Par exemple, la scène de début avec M. Picard et la Grande Z a lieu dans un cagibi minuscule et non ouvert au public attenant à l'église du musée. La scène finale face à la Directrice se tient, quant à elle, sur le palier d'un grand escalier de pierre poussiéreux, dérobé et caché au public, en fin d'après-midi entre chien et loup.

\section{Et techniquement, comment ça marche?}

Pour aider à résoudre les questions techniques posées, une riche documentation multimédia s'avérait nécessaire. Le petit écran du téléphone Nokia portable utilisé dans le précédent jeu ne convenait plus. Notre choix s'est porté sur l'iPhone qui, de plus, offre une interface plus intuitive. Cependant, les joueurs devaient pouvoir vérifier sur le terrain qu'ils avaient bien trouvé la salle ou l'objet recherché en lisant l'étiquette RFID associée. L'iPhone ne disposant pas de lecteur RFID, le téléphone portable Nokia restait donc indispensable pour localiser des joueurs dans le musée.

Les validations des salles et des objets permettaient de débloquer au fur et à mesure les questions du jeu ; un logiciel intermédiaire était nécessaire pour récolter ces informations, relier les différents matériels et 
faire progresser le jeu. Au final, ce logiciel était capable de faire transiter les informations entre les différents terminaux, gérer les aspects pervasifs du jeu : mobilité importante, traçabilité des joueurs par le Mâ̂tre du Jeu, mémorisation des différentes étapes de la progression des équipes dans le jeu et liaison avec le moteur d'adaptabilité. Il a été conçu à partir du moteur de jeux pervasifs uGASP (Pellerin, 2009).

Afin de maintenir les joueurs immergés dans l'histoire, il convenait de leur donner un rôle dans le scénario. Le contexte du jeu étant le passage d'épreuves pour intégrer une université, l'iPhone a été présenté comme le « cartable électronique » des candidats renfermant, outre les questions constitutives des épreuves, les différentes ressources documentaires nécessaires pour répondre, ainsi que les résultats obtenus. Toutes ces informations étaient communiquées lors de la scène introductive.

Le jeu utilisait un mobile lecteur RFID et un iPhone, double équipement qui a été bien accepté par les joueurs, comme les retours des premiers tests nous l'ont confirmé (Damala, 2010). Aujourd'hui ces deux technologies seraient combinées dans certains smartphones ou encore, les tags RFID pourraient être remplacés par des QR Code.

\section{Conclusions}

\section{Retours et bilans}

Pour ce second jeu, 4 sessions de tests ont été réalisées, regroupant une cinquantaine de personnes dans 16 équipes. Les joueurs avaient aussi bien une formation technique que sciences humaines. L'âge variait entre 20 et 55 ans. Leurs appréciations et impressions ont été recueillies via des questionnaires,

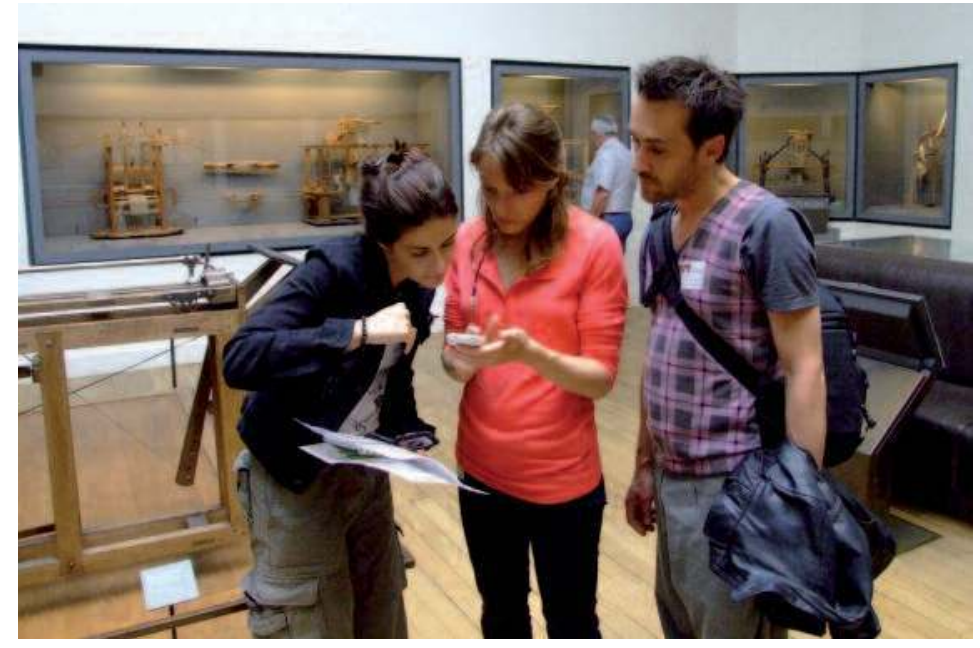

Les joueurs de PUPN sur le terrain ๑) PLUG des mini-interviews durant les sessions de jeu et spontanément en fin de session.

D'après les retours des joueurs, les objectifs pédagogiques fixés au départ semblent avoir été atteints. Le jeu PUPN a su équilibrer jeu et apprentissage. Les parcours des joueurs attestent que chaque équipe est allée au bout de sa quête sans aucun abandon ni découragement. Le moteur d'adaptabilité a joué un grand rôle dans cette acceptation du jeu et dans la persévérance dont les joueurs ont fait preuve : il a su maintenir l'intérêt des joueurs grâce à un processus d'apprentissage en douceur calé sur leur profil. La scène finale face à la Directrice a révélé les connaissances acquises par les équipes, et les a renforcées via le dialogue direct entre les « apprenants » et les «maittres ».

Toutefois, notre objectif n'a pas été pleinement atteint. En effet, par manque de temps, nous n'avons pas pu développer l'aspect historique autant que nous l'aurions souhaité. Les joueurs n'ont donc pas pu découvrir l'importance du contexte sociétal dans le processus de création et d'acceptation d'une innovation.

L'univers du jeu a contribué pour beaucoup à la motivation et l'implication des joueurs. L'immersion était essentielle pour qu'ils « rentrent » dans le jeu et se laissent emporter par l'histoire et les personnages. L'amusement, l'originalité des situations, les rebondissements dus aux joutes, aux indices supplémentaires ou aux interventions du Maître de Jeu, et enfin le but même de la mission ont aidé à rendre naturels et intuitifs l'avancée dans le jeu et l'apprentissage. Par ailleurs, d'un point de vue comportemental et sociologique, il a été instructif de noter l'attitude des joueurs face au commissaire de police lors de la dernière scène du jeu : ont-ils ou non dévoilé le secret de l'UPN au commissaire ? Dans la majorité des cas,

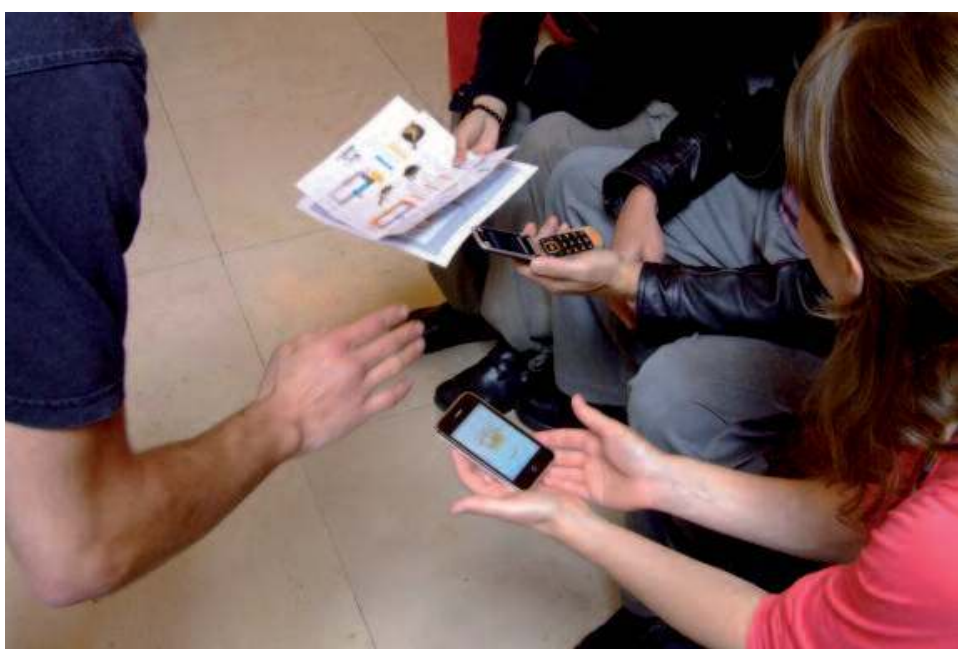

Les joueurs en pleine chasse au trésor ๑) PLUG 
non. Ce résultat est intéressant à plus d'un titre, car il révèle des personnalités et des prises de position réelles face à des situations virtuelles dans le cadre d'un environnement de jeu.

En ce qui concerne le moteur d'adaptabilité, outre son intérêt pour équilibrer la difficulté du jeu, c'est aussi un premier pas vers une meilleure connaissance du comportement des joueurs-visiteurs. Mais tracer des comportements ne suffit pas : il faut être capable de les analyser de façon automatique, afin de dégager des profils de visiteur-joueur, des caractéristiques de visite-jeu et d'alimenter ainsi le musée dans sa réflexion sur la médiation et sur ses collections. Ce type de fonctionnalité peut s'appliquer plus généralement aux visites sans aucun aspect jeu.

\section{Travaux futurs : ArtSense}

Les retours des sessions du jeu ont démontré le potentiel des applications multimédia comme une forme alternative de médiation culturelle et d'appropriation des concepts scientifiques abstraits de manière adaptée à des profils de visiteurs différents. Par ailleurs de nombreux musées et institutions culturelles investissent de plus en plus dans des technologies mobiles de pointe (comme par exemple le Mobile Learning Institute, une initiative du Smithsonian Institute à Washington regroupant 19 musées et galeries http://s301846506. onlinehome.fr/wp-content/uploads/2010/01/ 9rfidfosteringinteractions2.pdf).

Suivant cette conviction d'exploitation et de mise en valeur des collections par les technologies mobiles multimedia, le musée des Arts et Métiers et le CEDRIC participent et collaborent à nouveau dans le cadre du projet Européen ARtSENSE (www.artsense.eu) visant la conception et l'implémentation d'un guide de visite culturelle en Réalité Augmentée Adaptative. Le consortium, dont le chef de projet est le Forschungszentrum Informatik (FZI) de Karlsruhe, est formé de dix partenaires européens. La vraie particularité et originalité du projet est l'implication et la collaboration de professionnels du domaine du patrimoine culturel : le musée des Arts et Métiers, le museo nacional de Artes decorativas de Madrid et la Foundation for Art and Creative Technology à Liverpool, tous coordonnés par le CEDRIC qui par ailleurs assure la liaison de leurs travaux avec les partenaires techniques et industriels.

La nouvelle notion de Réalité Augmentée Adaptative propose d'aller plus loin dans l'expérience de la visite et de l'apprentissage assistés. Elle prévoit, premièrement, l'incrustation d'objets numériques dans l'environnement réel avec lequel le visiteur peut interagir, puis, la prise en compte des informations dans l'environnement acoustique, et enfin, une expérimentation avec des capteurs physiologiques qui, à long terme, pousseront plus loin notre compréhension de la physiologie de l'intérêt et, de manière plus globale, l'apprentissage dans le cadre d'une visite culturelle.

Les auteurs tiennent à remercier pour leur collaboration active : Armelle Prigent (enseignante à l'université de La Rochelle, responsable de la spécialité programmation du master Jeu Video et Médias Interactifs Numériques, directrice adjointe de l'ENJMIN, École nationale du Jeu vidéo et des médias Interactifs Numériques et membre du laboratoire L3I, laboratoire Informatique, Image et Interaction de l'université de La Rochelle), Nicolas Rempulski (Doctorant, laboratoire L31 de l'université de La Rochelle), Gautier de Hauteclocque (président de l'association Dune Aventures) et Sophie de Mareuil (membre de l'association Dune Aventures).

Note

(1) La théorie du flow recommande de garder l'engagement (le flow) dans un intervalle entre l'ennui et le stress.

\section{Bibliographie}

Astic, I. et Aunis, C. PLUG : les secrets du musée, La Lettre de l'OCIM $\mathrm{n}^{\circ} 125$, septembre-octobre 2009, pp. 5-11.

Astic, I., Aunis, C., Damala, A. et Gressier-Soudan, É. A Ubiquitous Mobile Edutainment Application for Learning Science through Play, in Trant, J. and Bearman, D. (eds) Museums and the Web 2011 : proceedings. Toronto : Archives \& Museum Informatics, 2011.

Csikszentmihalyi, M. Flow : The Psychology of Optimal Experience. London : Harper Perennial, 1990.

Damala, A., Astic, I. et Aunis, C. PLUG, Université Paris-Nuit : a Design Reiteration of a Mobile Museum Edutainment. The $11^{\text {th }}$ International Symposium on Virtual Reality, Archaeology and Intelligent Cultural Heritage, 2010, pp. 91-94.

Gentès, A., Jutant, C., Guyot-Mbodji, A. et Simatic, M. RFID Technology : Fostering humain interactions, IADIS International Conference Game and Entertainment Technologies 2009, Algarve, Portugal, pp. 67-14.

Pellerin, R. Contribution à l'ingénierie des jeux multijoueurs ubiquitaires. Mémoire de thèse, Cnam, 2009. 